Service social

\title{
Une expérience de service social des groupes dans un centre d'hébergement et de réadaptation sociale
}

\section{Pascal Dabouis}

Volume 46, numéro 2-3, 1997

Groupes - Symposium 1997

URI : https://id.erudit.org/iderudit/706772ar

DOI : https://doi.org/10.7202/706772ar

Aller au sommaire du numéro

Éditeur(s)

École de service social de l'Université Laval

ISSN

1708-1734 (numérique)

Découvrir la revue

Citer cet article

Dabouis, P. (1997). Une expérience de service social des groupes dans un centre d'hébergement et de réadaptation sociale. Service social, 46(2-3), 285-290.

https://doi.org/10.7202/706772ar d'utilisation que vous pouvez consulter en ligne. 


\section{Une expérience de service social des groupes dans un centre d'hébergement et de réadaptation sociale}

Pascal DABOUIS

\section{CONTEXTE}

Mon champ d'intervention sociale se situe dans le cadre d'un centre d'hébergement et de réadaptation sociale (CHRS) géré par une association, l'Entraide Ouvrière (89 salariés, 350 adhérents, 120 bénévoles). Cette association née dans l'après-guerre s'est donné pour but d'accueillir toute personne à la rue ou en grande difficulté sociale. Elle a une capacité d'hébergement de 250 lits. Elle intervient dans le champ de l'Aide sociale et plus particulièrement de l'Aide sociale à l'hébergement. Animée par de nombreux militants et bénévoles, elle a une mission de service public conventionné avec l'État. Ses objectifs de travail sont de permettre aux personnes accueillies d'accéder à leurs droits de citoyens et de les accompagner sur les voies d'une réinsertion et d'une autonomie sociale durables.

Ce public, hommes, femmes et familles, a très souvent connu des ruptures d'ordre familial, social et professionnel. Il est stigmatisé dans le chômage, l'exclusion sociale, la marginalité et la pauvreté.

Devant la massification de ces phénomènes, les politiques sociales ont répondu par des dispositifs complexes qui mettent souvent 
les individus dans l'assistance et la culpabilité. Le RMl est un bon exemple car, au vu de la situation sociale en France, soit la personne enclenche un processus de culpabilité s'il y a échec du contrat d'insertion, soit elle travaille à l'acceptation de sa condition d'exclu ou tout du moins de citoyen inutile et assisté.

Ne devrions-nous pas rechercher l'éclairage de ces logiques d'exclusion et de pauvreté dans le développement du libéralisme et des sacro-saintes lois du marché qui priment une prétendue économie rationnelle et pragmatique au détriment d'un développement social et culturel?

\section{MAIS QUEL EST LE PROBLÈME?}

Les personnes accueillies dans les CHRS de l'Entraide Ouvrière ont, durant le premier mois de "prise en charge ", une période de bilan appelée " 21 jours ». Celle-ci s'articule autour de quatre thèmes officialisés par la signature d'un contrat de séjour : la santé, les connaissances générales, le travail et l'histoire de vie. Ces évaluations s'effectuent au moyen d'entretiens individuels dans la logique de l'écoute, du conseil, du soutien et de la persuasion. Si elles permettent d'établir une relation privilégiée d'écoute importante, elles sont parfois confondues dangereusement avec une certaine pratique "psychologisante ". Le problème est qu'il est bien difficile de travailler l'aspect social dans le contexte d'une relation duale.

Les études sur le public en situation d'exclusion sociale font apparaître un isolement important (rapport du Centre d'étude des revenus et des coûts, Précarité et risque d'exclusion en France, Documentation française $n^{\circ} 109$ ). La pratique du travail psychosocial individualisé a tendance à renforcer cette difficulté. Le travail interactionnel avec le travailleur social ne suffit pas pour prendre en compte la dimension sociale de la personne, ne la resitue pas dans son contexte social. Cela ne l'aide pas à retisser ses liens sociaux nécessaires et à vivre sa citoyenneté.

L'expérience menée du travail social avec les groupes avait pour objectif de rompre avec l'individualisme de cette pratique, d'agir sur les phénomènes d'entraide mutuelle, de resituer l'acteur-citoyen et d'engager une recherche méthodologique permettant d'agir sur la socialisation et le sociétal. Relativement isolée dans la conviction 
de cette volonté de travail de groupe, la recherche devait prouver le préalable avancé, c'est-à-dire la « supériorité » du travail social avec les groupes, sa pertinence et son efficacité sur le travail individualisé avec des personnes en grande difficulté.

\section{MÉTHODE DE DÉMONSTRATION PRÉALABLE}

L'expérience a commencé par la mise en place de deux groupes que j'avais la charge d'animer dans la période d'évaluation de 21 jours. Le groupe A était constitué de dix personnes rencontrées exclusivement par des entretiens individuels. Quant au groupe B, il était divisé en deux sous-groupes de cinq personnes avec réunion collective et entretien individuel une fois par semaine. Pour comparer les deux méthodes, j'ai proposé un tableau d'évaluation comparative sur l'activité des 21 jours. Trois axes de critères ont été ici pris en considération.

\section{Comparaison chiffrée}

Le travail de groupe allait-il atténuer les difficultés et l'appréhension de la période de 21 jours?

Participation

Groupe A : 5 personnes sont parties avant la fin des 21 jours. Groupe B : 7 personnes ont participé à toutes les réunions.

Signature du contrat de séjour

Groupe A : 5 contrats signés

Groupe B : 7 contrats signés

\section{Accès aux droits sociaux}

Le groupe permettait-il d'accéder plus rapidement aux droits sociaux - santé, revenu, logement, emploi, relation? II s'est avéré que le travail de groupe n'apparaissait pas plus pertinent pour l'accès aux droits. La logique des dispositifs sociaux n'intègre que la dimension individuelle et peut par conséquent pondérer ce résultat.

\section{Analyse des réunions sur l'entraide}

Une évaluation était ici impossible, car cela aurait demandé la mise en place de critères extrêmement complexes et sujets à interprétation 
tendancielle en raison du petit nombre de personnes concernées. Cependant, la dynamique de groupe qui apparaissait dans les stratégies de resocialisation demandait à être confirmée.

De ce premier bilan, la tendance de l'efficacité du travail de groupe se dégageait suffisamment pour passer à une période plus longue et à une organisation de travail de groupe plus approfondie. L'équipe y était plus disponible.

\section{COMPARAISON SUR SIX MOIS}

Nous sommes passés à une comparaison sur six mois avec la mise en place d'un travail de groupe en systématique sur la période 21 jours et les résultats de l'année antérieure où nous ne travaillions qu'en entretien individuel.

Les deux périodes retenues furent janvier à juin 1993 et janvier à juin 1994.

\section{Comparaison chiffrée}

Nous avons accueilli 113 personnes en 1993 et 116 en 1994. La différence de trois personnes nous permet donc une comparaison équilibrée.

\section{Participation}

Année 1993 : sur 113 personnes dans les 21 jours, 43 ont suivi le cycle jusqu'à son terme, soit $38 \%$.

Il fallait compter deux convocations, orales ou écrites, pour un entretien réalisé, soit $50 \%$ de déperdition.

Année 1994 : sur 116 personnes nous avons eu un taux de participation aux réunions de $84 \%$. Les personnes continuaient à être vues individuellement pour ce qui concerne leur dossier personnel.

Signature des contrats de séjour

Année 1993 : 43, soit $38 \%$

Année 1994 : 68 , soit $58 \%$ 
On note plus de $20 \%$ de différence, soit une augmentation de $36,7 \%$ pour l'année 1994. Les réunions collectives avaient donc un effet plus attractif que les entretiens individuels.

\section{Accès aux droits sociaux}

Comme lors de la première expérience, il est apparu qu'en termes de droits sociaux la méthode de travail social avec les groupes ne semblait pas plus adéquate que le travail individuel. Globalement, les personnes n'accèdent pas plus vite à leurs droits.

Cependant, la mise en commun des difficultés dans le groupe favorise des relations d'entraide et d'acceptation des problèmes. II est plus intéressant de traiter en groupe les difficultés qui surviennent dans la résolution des problèmes d'ordre administratif, car on peut alors souvent démontrer que la personne n'est pas seule et isolée dans ses difficultés. Le groupe permet de relativiser la parole, perçue parfois comme toute-puissante, du travailleur social.

II serait inopportun de vouloir prouver que le groupe accélère les réinsertions,

car le fait d'obtenir un emploi ou de ne pas en trouver dans un contexte actuellement peu réjouissant ne dépend que trop peu de l'activité des travailleurs sociaux. Par contre ils peuvent être évalués sur la manière dont ils auront optimisé les chances pour les personnes de s'insérer... (Le Poultier, Recherches évaluatives en travail social, PUG).

\section{Analyse des réunions}

Dans ce type de groupe, le travailleur social est toujours resté l'animateur, car le croisement des difficultés des personnes - toxicomanie, alcoolisme, psychiatrie, jeunes en galère, marginaux, RMlstes, etc. - demande une attention permanente à l'équilibre de la vie du groupe.

L'homogénéité du groupe s'articulait sur l'expérience des membres de l'évaluation des 21 jours. La mise en commun des difficultés permettait de baisser les angoisses liées à la peur de l'échec, de confronter ses expériences et ses difficultés, de s'interpeller sur les productions. De ces confrontations ont jailli des sujets tels que le sida, l'alcool, la marginalité, les problèmes de la vie collective. Tous 
les sujets provoquaient des débats riches et animés, car ils s'appuyaient sur le vécu des membres du groupe. Des conflits s'exprimaient lorsque la composition du groupe était propice à leurs apparitions : écart d'âge important, personne alcoolisée, personne ayant des troubles psychologiques, personne très marginalisée.

Le débat et la parole sont précieux, mais ils peuvent être limités quant à l'incidence qu'ils ont sur l'individu. C'est au travers d'expériences et de construits communs que cette forme d'intervention est apparue la plus intéressante.

Cela s'est concrétisé par les phénomènes d'entraide mutuelle. Celle-ci passait par l'accompagnement d'un résidant par un autre à l'Agence nationale pour l'emploi, à la Caisse d'allocations familiales, aux ateliers de l'association ou par une évaluation commune des connaissances générales. Nous avons constaté un net changement des comportements à la suite de la mise en place du travail de groupe. Certains se situaient autour du droit au logement, de marches de chômeurs.

Cette expérience a effectivement démontré l'intérêt et l'aspect incontournable du travail social avec les groupes. La démonstration fut validée par l'équipe. Mais elle ne fut pas suffisante en tant que telle car, si l'idée est partagée, le manque de formation sur la conduite des groupes et ses dynamiques, linquiétude que provoque le groupe composé de personnes en difficulté ont été de véritables freins à son élargissement.

\section{CONCLUSION}

L'intervention du travail social avec les groupes ne situe pas seulement l'individu dans sa dimension singulière, mais en tant qu'être dans sa dimension sociale. La promotion des groupes permet à l'individu de retrouver un rôle et une identité sociale. Elle s'oppose à une démarche d'assistance.

Pascal DABOUIS

Animateur, adjoint chef de service Association Entraide Ouvrière Tours, France 